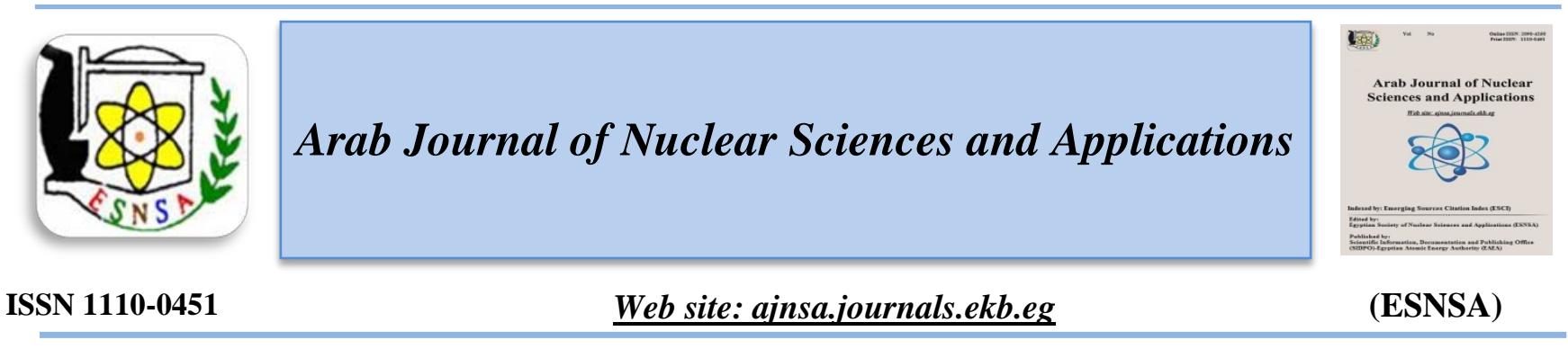

\title{
Determination of Uranium Isotopic Ratios by Alpha Spectrometry for Nuclear Materials Control Purposes
}

\author{
Raed Abouzeid ${ }^{1}$, Hany Khedr ${ }^{1}$, Ahmed El-sharkawy ${ }^{1}$, Omnia Ali $^{2}$ and Abdel hakim Kandil ${ }^{2}$ \\ ${ }^{(1)}$ Egyptian Nuclear and Radiological Regulatory Authority, Cairo, Egypt \\ ${ }^{(2)}$ Chemistry Department, Faculty of science, Helwan University, Cairo, Egypt
}

Received $31^{\text {st }}$ Jan. 2020 Uranium is the most common element in the nuclear fuel cycle. Different techniques have been used for Accepted $12^{\text {th }}$ May analyzing the isotopic composition of uranium. Precise determination of uranium isotopic ratios for an 2020 efficient control of nuclear material was carried out through the modification of the used chromatographic method of uranium. Alpha spectrometry was utilized to identify different types of natural, depleted and enriched samples for safeguard purposes. Uranium element was extracted from other interfering radionuclides, purified, electrodeposited on a stainless-steel disc, and measured using alpha-spectrometer, then isotopic ratios were calculated. For comparison purposes, the samples were also measured using a hyper pure germanium (HPGe) spectrometer and the uranium isotopic ratios were obtained. The results obtained by both techniques were in agreement within difference ranged from -8.7 to $12 \%$. It was concluded that alpha spectrometric measurements of uranium isotopes preceded by efficient radiochemical separation using extraction chromatography is a fast and reliable technique for safeguard purposes.

Keywords: Safeguards / Uranium Isotopic Ratio / Gamma Spectrometry / Extraction Chromatography / Alpha Spectrometry

\section{Introduction}

Natural uranium is a mixture of the three radioactive isotopes: ${ }^{238} \mathrm{U}$, ${ }^{235} \mathrm{U}$ and ${ }^{234} \mathrm{U}$ with abundance of $99.2742 \%, 0.7204 \%$ and $0.0054 \%$ respectively [1, 2]. Uranium has achieved its significance in civil nuclear technology (nuclear power plants, research reactors) because of its fissile isotope ${ }^{235} \mathrm{U}$. The ${ }^{235} \mathrm{U} /{ }^{238} \mathrm{U}$ can be used for distinguishing the natural sources of uranium from the artificial ones [3]. The uranium that remains after the enrichment process is commonly called Depleted Uranium (DU). The importance of the isotopic composition of uranium for safeguards purposes has initiated the need to be able to apply fast and reliable methods for the uranium enrichment determination. Two major techniques are widely used for the determination of the isotopic composition of uranium, gamma spectrometry and alpha spectrometry. For establishing nuclear safeguards and for nuclear forensic to prevent illicit trafficking on nuclear materials, some previous studies discussed the use of high resolution gamma spectrometry to determine the total U content, isotopic content, and the enrichment of ${ }^{235} \mathrm{U}$ to date the time of purification and/or enrichment of uranium samples using some commercial analytical software codes [4]. Other studies developed useful methods to measure the uranium enrichment of samples with ${ }^{238} \mathrm{U}$ activity difficult to be measured as a result of lack of secular equilibrium or high enrichment [5]. Determination of uranium in different matrices by

Corresponding author: raed.abouzeid1986@gmail.com

DOI: 10.21608/ ajnsa.2020.21370.1324

(C) Scientific Information, Documentation and Publishing Office (SIDPO)-EAEA 
alpha spectrometry requires several radiochemical procedures to separate these radionuclides from the matrix [6]. Radiochemical separation method was described and developed by Macsik et al. [7] for the determination of uranium and some other actinides in safeguards swipe samples. The aim of this work was to achieve highly accurate and reliable results for the determination of the ${ }^{234} \mathrm{U} /{ }^{238} \mathrm{U}$ and ${ }^{235} \mathrm{U} /{ }^{238} \mathrm{U}$ activity ratios in safeguarded materials using selective extraction chromatography and alpha spectrometry.

\section{Materials and Methods}

Materials and reagents

All chemicals used in this work were of analytical grade unless otherwise indicated. All references to water refer to Double Deionized Water (DDW).

Three sets of solid samples represent the three categories of uranium [Natural (NU), DU and Enriched (EU)] were used in this study ( Table 1).

\section{Apparatus and procedures}

The gamma measurements were carried out using a Hyper Pure Germanium (HPGe) detector (Canberra, falcon $5000^{\circledR}$, USA) with a relative efficiency of $18 \%$, with the associated electronics and Multi-Channel Analyzer (MCA) [8]. Each sample was measured for 1 hour, directly attached to the detector. The isotopic ratios were calculated using the MGAU code [9], which depends on both gamma and $X$ rays radiation in the range from 84 $\mathrm{keV}$ to $130 \mathrm{keV}$. After that, and for alpha measurements, samples were dry- ashed at $400{ }^{\circ} \mathrm{C}$ for 6 hours using muffle furnace, then appropriate quantities were further digested using a closed pressurized high throughput microwave digestion/extraction/synthesis workstation (Shanghai Sineo Microwave Chemistry Technology Co., Ltd. MASTER-15, China [10].

Pre-packed extraction chromatography resin columns UTEVA (2ml with particle size 100-150 $\mu \mathrm{m})$, supplied by Triskem International Co. (France), were used in the extraction and purification of uranium following Eichrom method [11]with some modifications. The alpha sources were prepared using two posts electrodeposition device (SDEC EDP9002, France) by the electrodeposition on a stainless-steel disk. The alpha measurements were carried out using ORTEC Ultra ${ }^{\mathrm{TM}}$ ENS ion-implanted silicon detector (USA) of a $450 \mathrm{~mm}^{2}$ active area. Typical measuring life-time of samples ranged from 5400 to 86400 seconds depending on the activity of the sample. The efficiency calibration was carried out using a standard alpha multi source supplied by Eckert \& Ziegler with total uncertainty of $\pm 3 \%$.

Table (1): The different types of samples and their chemical composition

\begin{tabular}{ccc}
\hline Sample Code & Type & $\begin{array}{c}\text { Chemical } \\
\text { composition }\end{array}$ \\
\hline NU1 & Natural & $\mathrm{UF}_{4}$ \\
NU2 & Natural & Ammonium uranate \\
NU3 & Natural & Ammonium uranate \\
NU4 & Natural & $\mathrm{UO}_{3}$ \\
NU5 & Natural & $\mathrm{UO}_{3}$ \\
NU6 & Natural & $\mathrm{UO}_{3}$ \\
DU1 & Depleted & $\mathrm{Uranyl} \mathrm{nitrate}$ \\
DU2 & Depleted & $\mathrm{Scrap}$ \\
DU3 & Depleted & $\mathrm{UO}_{2}$ \\
EU1 & Enriched & $\mathrm{UO}_{2}$ \\
EU2 & Enriched & $\mathrm{UO}_{2}$ \\
EU3 & Enriched & $\mathrm{UO}_{2}$ \\
EU4 & Enriched & $\mathrm{U}_{3} \mathrm{O}_{8}$
\end{tabular}

All measurements were carried out at the Egyptian Nuclear and Radiological Regulatory Authority (ENRRA) and all samples were provided by ENRRA. For samples digestion, instead of leaching the samples using total acids dissolution technique with concentrated acids, the digestion achieved using a closed pressurized microwave digestion system. Microwave digestion in closed vessels causes evaporation of gases and digestive acids (5 $\mathrm{ml}$ of each $\mathrm{HNO}_{3}(65 \%), \mathrm{HCl}(37 \%)$ and $\mathrm{HF}(40 \%)$ ) resulting in raising the pressure in the reaction vessel leading to more efficient digestion. Sample solution resulting from the previous microwave digestion step was evaporated, had additional treatment with nitric acid and $\mathrm{H}_{2} \mathrm{O}_{2}$ and dissolved in $10 \mathrm{ml} 3 \mathrm{M} \mathrm{HNO}_{3} / 1 \mathrm{M} \mathrm{Al}\left(\mathrm{NO}_{3}\right)_{3}, 2 \mathrm{~mL}$ of $0.6 \mathrm{M}$ ferrous sulfamate was added to reduce the actinides species to the (III) oxidation state, $1 \mathrm{~mL}$ of ascorbic acid was added to reduce any iron III species and the solution was left for few minutes. The sample solution was loaded on the UTEVA resin [12]. Uranium was eluted from the UTEVA column using $0.01 \mathrm{M} \mathrm{HCl}$, transferred to Teflon beaker [13]. During the previous treatment with different strong acids, the resin may bleed small 
quantities of the organic solid support of the extractant. Thus, after the elution of uranium from the UTEVA column, the uranium solution collected was evaporated to dryness and treated with $1 \mathrm{~mL}$ conc. $\mathrm{H}_{2} \mathrm{SO}_{4}$ and heated to dryness then $1 \mathrm{~mL} \mathrm{H}_{2} \mathrm{O}_{2}$ (30\% vol.) and $2 \mathrm{~mL}$ conc. $\mathrm{HNO}_{3}$ was added and heated until dryness three successive times for complete destruction of the organic matter [14]. Using 4\% ammonium oxalate and $1 \mathrm{M}$ $\mathrm{HCl}$ as electrolyte, samples were electrodeposited on stainless steel disks [15]. After the electrodeposition step, the disks were flamed to volatilize the gaseous radon daughters, and remove any organic impurities. Finally, the dry disks were counted using the alpha spectrometer. The background counts were determined using the same region of interest as those used for the samples and subtracted.

\section{Quality assurance}

Blank samples were measured in identical conditions for background estimation. Certified reference materials were analyzed using the same analysis procedure and the accuracy was determined. Errors were propagated due to peak area counting statistics, standards activity and weight [16]. The minimum detectable activity was determined based on the equation presented by Currie [17]. Repeated analyses and measurements of some selected samples and certified reference materials were carried out to assess the accuracy, precision and reliability of the method and data. Also, the accuracy and reliability of the radiochemical procedure were confirmed by the participation in the IAEA proficiency tests [18].

\section{Results and Discussion}

\section{Alpha spectrometry}

Natural, depleted and enriched uranium safeguarded samples were measured by alpha spectrometry. The alpha spectra showed a good resolution with full width at half maximum (FWHM) ranged from $20 \mathrm{keV}$ to $30 \mathrm{keV}$. Table (2) presents the activity concentrations of the uranium isotopes and the activity ratios obtained by alpha spectrometry. The associated combined uncertainties ranged from 3.3\% to 5.7\%.

As shown in Table (1), the ${ }^{235} \mathrm{U} /{ }^{238} \mathrm{U}$ activity ratios in the natural samples NU1 to NU6 had an average of 0.0443 , which is in agreement with the value of 0.046 for natural uranium. The ${ }^{234} \mathrm{U} /{ }^{238} \mathrm{U}$ activity ratios showed an average of 1.03. For the depleted samples (DU1, DU2, DU3), the ${ }^{235} \mathrm{U} /{ }^{238} \mathrm{U}$ activity ratios had an average of 0.028 while the ${ }^{234} \mathrm{U} /{ }^{238} \mathrm{U}$ activity ratios showed an average of 0.57 . For the enriched samples, EU1, EU2 and EU3 showed an average ${ }^{235} \mathrm{U} /{ }^{238} \mathrm{U}$ activity ratio of 0.705 , which is considered low enrichment, with an average enrichment percentage of $9.85 \%$. Sample EU4 showed ${ }^{235} \mathrm{U} /{ }^{238} \mathrm{U}$ activity ratio of 1.5 , with enrichment percentage of $18.87 \%$. Figures (1, 2 and 3) present examples of the alpha spectra of each type of the analyzed samples processed using the radiochemical separation procedure. The ${ }^{235} \mathrm{U}$ counts were corrected according to the intensity of the alpha peaks (4.3 MeV and 4.5 MeV).

\section{Gamma spectrometry}

The three sets of samples were measured by a HPGe detector. The ${ }^{235} \mathrm{U} /{ }^{238} \mathrm{U}$ activity ratios were also determined and presented with the associated $1 \sigma$ uncertainty in Table (3). Fig. (4) presents gamma spectrum of sample EU4.

A comparison between gamma and alpha
spectrometry results
${ }^{235} \mathrm{U} /{ }^{238} \mathrm{U}$ activity ratios, calculated from the two techniques, show a high agreement, as shown in Table (4).

\section{Conclusion}

Depleted, natural and low enriched safeguarded samples were analyzed for the $234 \mathrm{U} / 238 \mathrm{U}$ and 235U/238U activity ratios utilizing two techniques for the measurements; alpha and gamma spectrometry. The alpha spectra showed a good resolution with FWHM ranged from $20 \mathrm{keV}$ to 30 $\mathrm{keV}$, which is dependent on the extraction chromatography, the purification of uranium from interfering elements and the efficient preparation of the alpha source. The gamma results were in a good agreement with the alpha results within difference ranged from -8.7 to $12 \%$. The radiochemical separation methodology was found to be rapid and highly effective for isotopic ratio analysis by alpha spectrometry. The method is successfully applied to the safeguarded nuclear material of different enrichment ratios for uranium isotopic ratios. 
Table (2): Uranium isotopes activity concentrations (Bq), the activity ratios, and enrichment for alpha spectrometry

\begin{tabular}{cc|c|c|cccc}
\hline Code & ${ }^{238} \mathrm{U}(\mathrm{Bq})$ & ${ }^{235} \mathrm{U}(\mathrm{Bq})$ & ${ }^{234} \mathrm{U}(\mathrm{Bq})$ & ${ }^{235} \mathrm{U} /{ }^{238} \mathrm{U}$ & ${ }^{234} \mathrm{U} /{ }^{238} \mathrm{U}$ & Enrichment \%* & $\pm \%$ \\
\hline NU1 & 2.632 & 0.118 & 2.813 & 0.045 & 1.069 & 0.689 & 4.3 \\
$\mathrm{NU} 2$ & 0.687 & 0.031 & 0.681 & 0.045 & 0.991 & 0.696 & 5.1 \\
$\mathrm{NU3}$ & 0.376 & 0.015 & 0.381 & 0.040 & 1.011 & 0.624 & 3.7 \\
NU4 & 0.605 & 0.027 & 0.666 & 0.045 & 1.101 & 0.687 & 4.4 \\
NU5 & 0.217 & 0.010 & 0.227 & 0.044 & 1.045 & 0.678 & 5.5 \\
NU6 & 0.301 & 0.014 & 0.298 & 0.047 & 0.991 & 0.719 & 4.8 \\
& & & & & & & \\
DU1 & 0.227 & 0.009 & 0.181 & 0.039 & 0.797 & 0.598 & 3.3 \\
DU2 & 0.339 & 0.007 & 0.164 & 0.022 & 0.485 & 0.329 & 4.9 \\
DU3 & 0.402 & 0.010 & 0.177 & 0.025 & 0.439 & 0.383 & 3.6 \\
& & & & & & & 5.25 \\
EU1 & 0.069 & 0.051 & 1.033 & 0.738 & 15.057 & 10.256 & 5.2 \\
EU2 & 0.307 & 0.201 & 4.595 & 0.655 & 14.990 & 9.212 & 5.7 \\
EU3 & 0.186 & 0.135 & 2.729 & 0.724 & 14.690 & 10.075 & 4.8 \\
EU4 & 0.195 & 0.292 & 8.135 & 1.495 & 41.697 & 18.877 & 4.2 \\
\hline
\end{tabular}

* Mass is calculated via the equation (Activity $=$ Specific activity $\times$ mass $)$, and Enrichment $=\left({ }^{235} \mathrm{U}\right.$ mass $/$ $\left({ }^{235} U+{ }^{238} U+{ }^{234} U\right)$ masses $) \times 100$

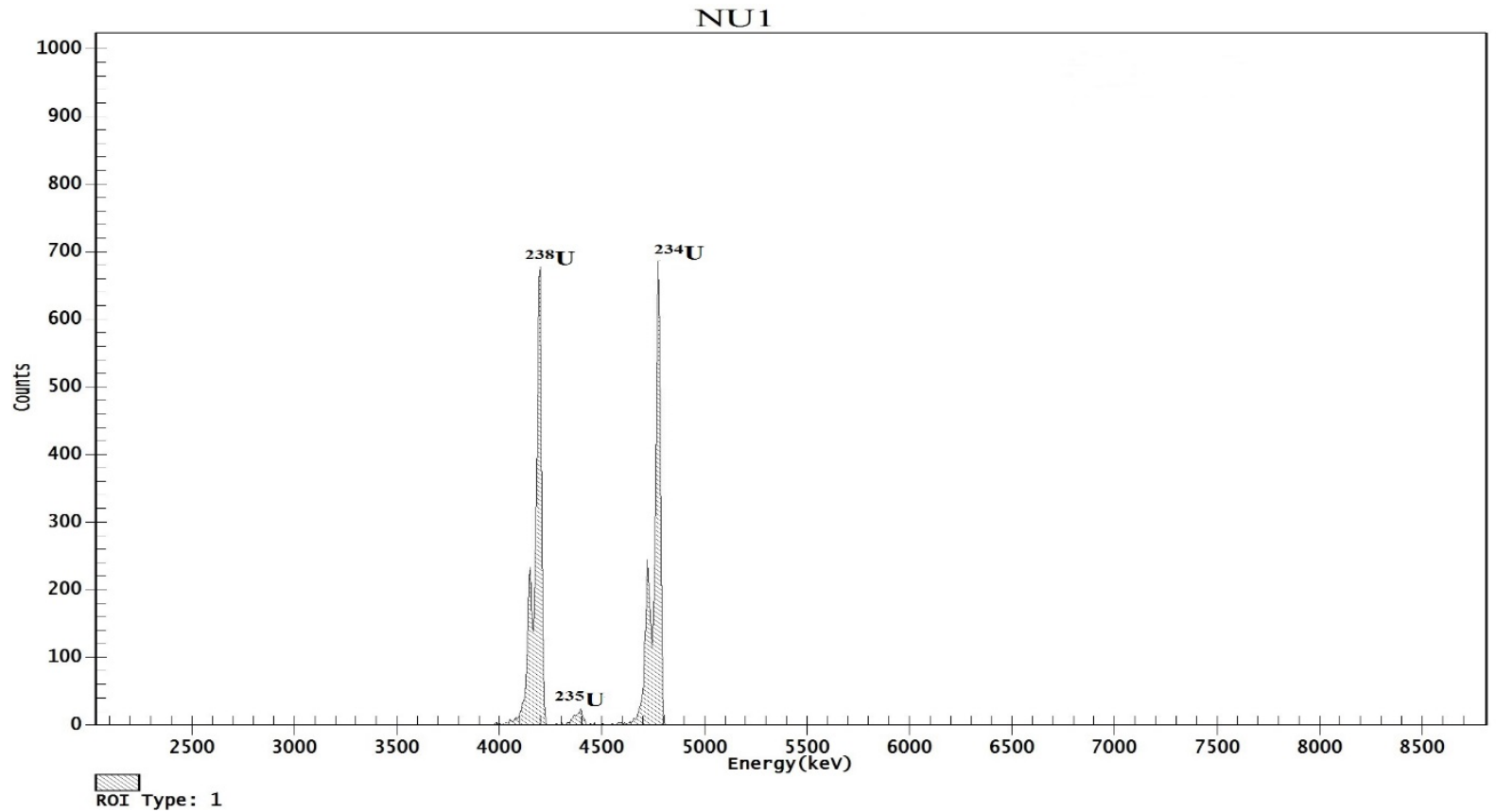

Fig. (1): The alpha spectrum of sample NU1 
DU2

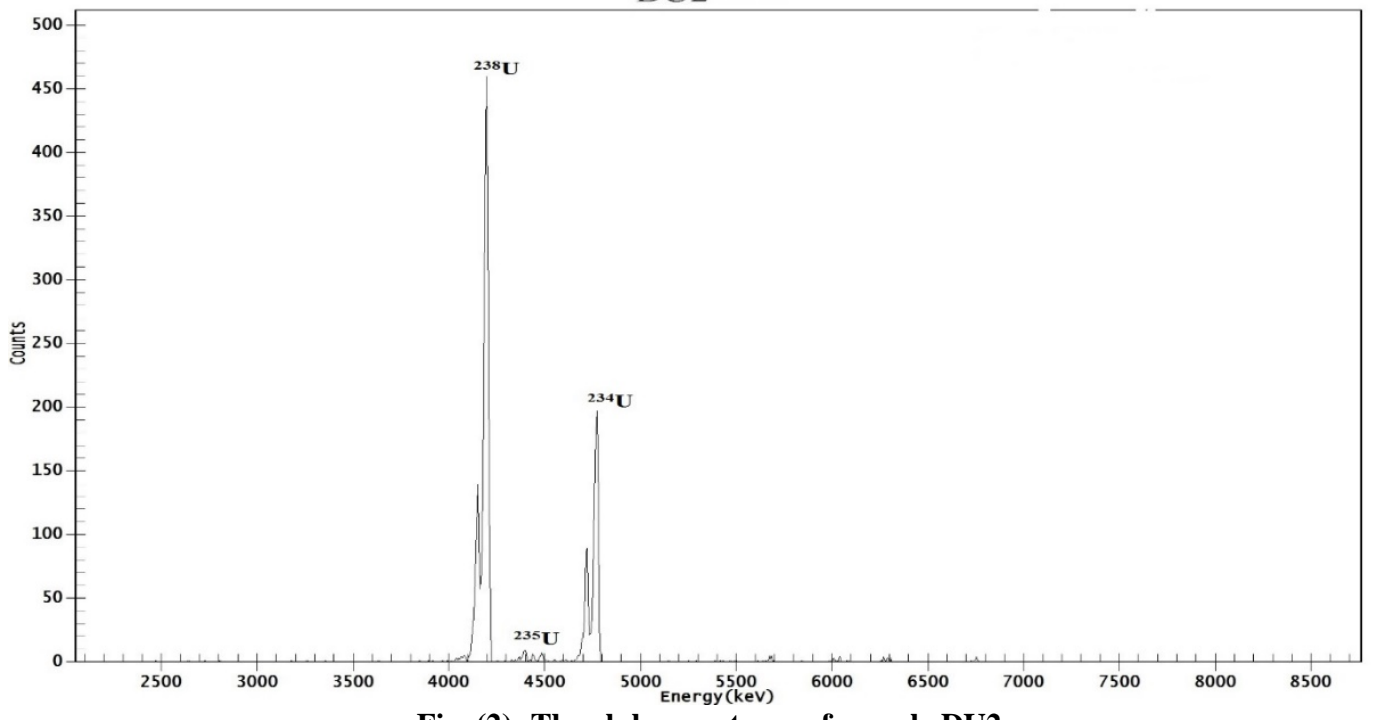

Fig. (2): The alpha spectrum of sample DU2

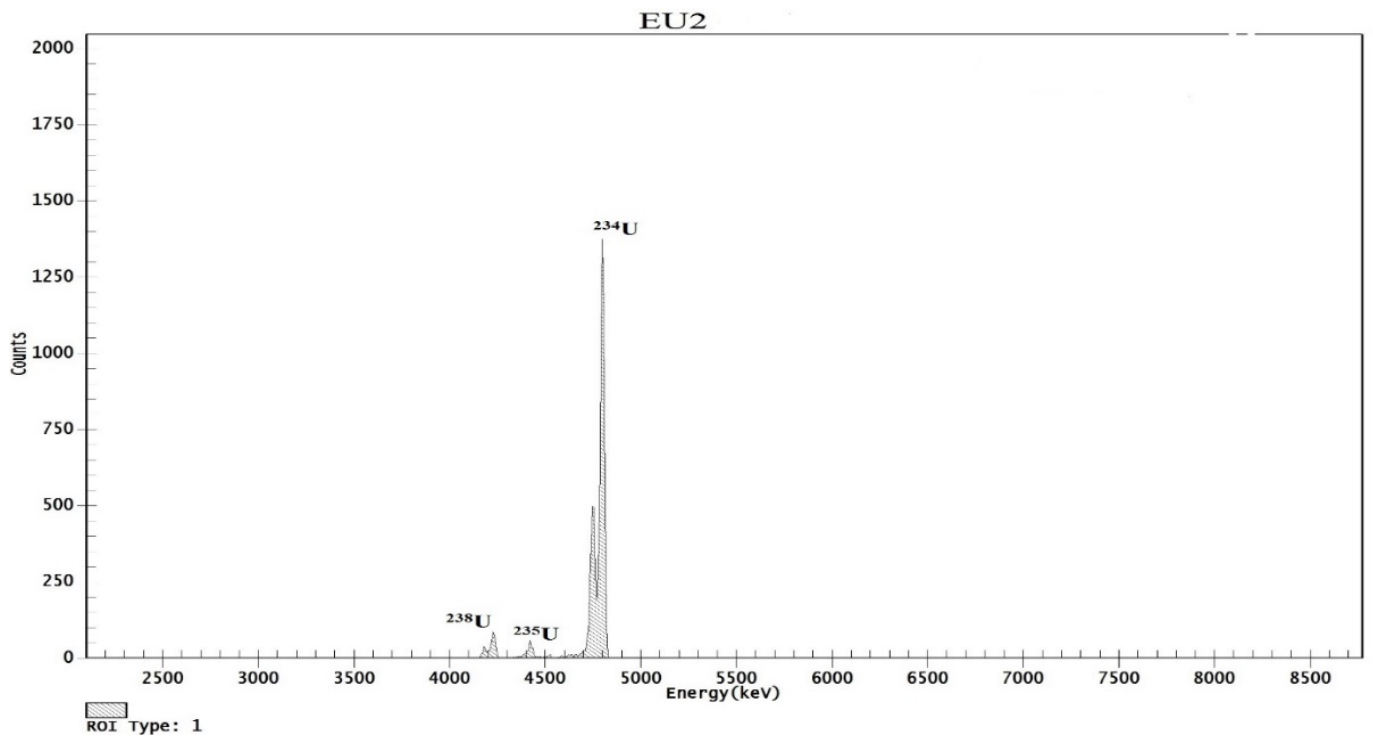

Fig. (3): The alpha spectrum of sample EU2

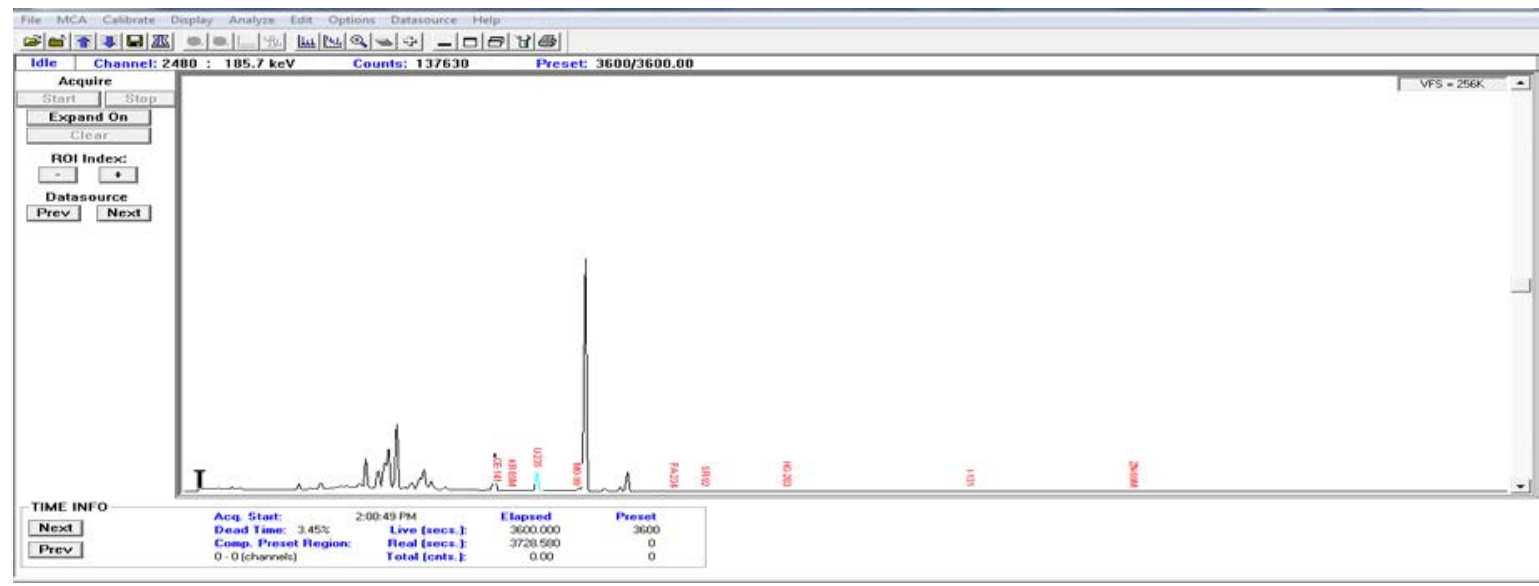

Fig. (4): The gamma spectrum of sample EU4 
Table (3): The MGAU code results weight $\%$ and ${ }^{235} U{ }^{238} U$ activity ratios for the three sets of samples

\begin{tabular}{lccc|c|c}
\hline \multicolumn{2}{c}{ MGAU Results abundance (weight\%) } & \multicolumn{3}{c}{ Activity* } \\
\hline Code & ${ }^{234} \mathrm{U}$ & ${ }^{23} \mathrm{U}$ & ${ }^{238} \mathrm{U}$ & ${ }^{235} \mathrm{U} /{ }^{238} \mathrm{U}$ & $\pm \%$ \\
NU1 & 0.0050 & 0.692 & 99.303 & 0.045 & 6.4 \\
NU2 & 0.0044 & 0.695 & 99.300 & 0.045 & 6.5 \\
NU3 & 0.0041 & 0.648 & 99.348 & 0.042 & 5.3 \\
NU4 & 0.0038 & 0.710 & 99.286 & 0.046 & 6.5 \\
NU5 & 0.0049 & 0.709 & 99.285 & 0.046 & 6.6 \\
NU6 & 0.0047 & 0.750 & 99.244 & 0.049 & 6.9 \\
& & & & 9.3 \\
DU1 & 0.0055 & 0.599 & 99.394 & 0.039 & 9.0 \\
DU2 & 0.0007 & 0.384 & 99.615 & 0.025 & 4.6 \\
DU3 & 0.0025 & 0.356 & 99.640 & 0.023 & 0.8 \\
EU1 & & & & & 1.6 \\
EU2 & 0.0773 & 9.594 & 90.328 & 0.685 & 0.716 \\
EU3 & 0.0758 & 9.983 & 89.917 & 0.718 & 0.7 \\
EU4 & 0.1956 & 10.00 & 80.293 & 1.568 & \\
\hline
\end{tabular}

* Activity is calculated via the equation (Activity = Specific activity $\times$ mass).

Table (4): Comparison between ${ }^{235} \mathrm{U} /{ }^{238} \mathrm{U}$ activity ratios from the two techniques

\begin{tabular}{llcc}
\hline Code & ${ }^{235} \mathbf{U}{ }^{238} \mathbf{U}$ by gamma & ${ }^{235} \mathbf{U} /{ }^{238} \mathbf{b y}$ alpha & difference \%* \\
\hline NU1 & $\mathbf{0 . 0 4 5}$ & $\mathbf{0 . 0 4 5}$ & 0.0 \\
NU2 & $\mathbf{0 . 0 4 5}$ & 0.045 & 0.0 \\
NU3 & $\mathbf{0 . 0 4 2}$ & 0.04 & 4.8 \\
NU4 & $\mathbf{0 . 0 4 6}$ & 0.045 & 2.2 \\
NU5 & $\mathbf{0 . 0 4 6}$ & 0.044 & 4.3 \\
NU6 & $\mathbf{0 . 0 4 9}$ & 0.047 & 4.1 \\
& & & 0.0 \\
DU1 & $\mathbf{0 . 0 3 9}$ & 0.039 & 12.0 \\
DU2 & $\mathbf{0 . 0 2 5}$ & 0.022 & -8.7 \\
DU3 & $\mathbf{0 . 0 2 3}$ & 0.025 & \\
& & & -7.7 \\
EU1 & $\mathbf{0 . 6 8 5}$ & 0.738 & 8.5 \\
EU2 & $\mathbf{0 . 7 1 6}$ & 0.655 & -0.8 \\
EU3 & $\mathbf{0 . 7 1 8}$ & 0.724 & 4.7 \\
EU4 & $\mathbf{1 . 5 6 8}$ & 1.495 & \\
\hline
\end{tabular}

$*$ difference $\%=\frac{\text { gamma result-alpha result }}{\text { gamma result }} \times 100$ 


\section{References}

1. "IAEA Safeguards Glossary, Edition 2001, international nuclear verification series No. 3," IAEA, Austria. [Online]. Available: https://www.iaea.org/sites/default/files/iaea_sa feguards_glossary.pdf.

2. A. Luca, "Experimental determination of the Uranium enrichment ratio,” Rom. Jour. Phys, vol. 53, no. 1-2, pp. 35-39, 2008.

3. M. Berglund and M. E. Wieser, "Isotopic compositions of the elements 2009 (IUPAC Technical Report),” Pure Appl. Chem., vol. 83, no. 2, pp. 397-410, 2011.

4. H.-D. Choi and J. Kim, "Basic characterization of uranium by high-resolution gamma spectroscopy,” Nucl. Eng. Technol., vol. 50, no. 6, pp. 929-936, 2018.

5. S.-H. Kim, J.-Y. Song, J.-Y. Jeon, J.-Y. Choi, and H. Seo, "Feasibility study on evaluating uranium enrichment with the High Resolution Gamma-Ray Spectrometry and X-ray fluorescence,” Appl. Radiat. Isot., vol. 148, pp. 132-137, 2019.

6. M. H. Lee, E. C. Jung, W. H. Kim, and K. Y. Jee, "Sequential separation of the actinides in environmental and radioactive waste samples," J. Alloys Compd., vol. 444, pp. 544-549, 2007.

7. Z. Macsik et al., "Improved radioanalytical method for the simultaneous determination of Th, U, Np, Pu and Am (Cm) on a single TRU column by alpha spectrometry and ICP-MS," Radiochim. Acta Int. J. Chem. Asp. Nucl. Sci. Technol., vol. 101, no. 4, pp. 241-252, 2013.

8. S. A. Dewji et al., "Validation of gamma-ray detection techniques for safeguards monitoring at natural uranium conversion facilities,” Nucl. Instruments Methods Phys. Res. Sect. A Accel. Spectrometers, Detect. Assoc. Equip., vol. 823, pp. 135-148, 2016.

9. R. Gunnink et al., "MGAU: A new analysis code for measuring \{sup 235\} U enrichments in arbitrary samples," Lawrence Livermore National Lab., CA (United States), 1994.

10. H. S. Amoli, J. Barker, and A. Flowers, "Closed vessels microwave digestion method for uranium analysis of soils using alphaspectroscopy,” J. Radioanal. Nucl. Chem., vol. 273, no. 2, pp. 281-284, 2007.

11. "Eichrom Technologies, Inc., Uranium in Soil (2 grams Samples). Analytical Procedures, ACS07, Rev. 1.6,” 2014. [Online]. Available: https://www.eichrom.com/wpcontent/uploads/2018/02/acs07-16_uraniumin-soil.pdf.

12. A. M. El-Sharkawy, " $234 U / 238 U$ activity ratios in groundwaters from two aquifers in Saudi Arabia, and correlation with water chemistry,” J. Radiat. Res. Appl. Sci., vol. 11, no. 4, pp. 368-372, 2018.

13. L. Popov, "Method for determination of uranium isotopes in environmental samples by liquid-liquid extraction with triisooctylamine/xylene in hydrochloric media and alpha spectrometry,” Appl. Radiat. Isot., vol. 70, no. 10, pp. 2370-2376, 2012.

14. S. L. Maxwell, B. K. Culligan, and G. W. Noyes, "Rapid separation method for actinides in emergency soil samples,” Radiochim. Acta Int. J. Chem. Asp. Nucl. Sci. Technol., vol. 98, no. 12, pp. 793-800, 2010.

15. K. W. Puphal and D. R. Olsen, "Electrodeposition of. alpha.-emitting nuclides from a mixed oxalate-chloride electrolyte," Anal. Chem., vol. 44, no. 2, pp. 284-289, 1972.

16. G. de Silva, "Uncertainty of Analytical Determinations,” J. AOAC Int., vol. 86, no. 5, pp. 1077-1083, 2003.

17. L. A. Currie, "Limits for qualitative detection and quantitative determination. Application to radiochemistry," Anal. Chem., vol. 40, no. 3, pp. 586-593, 1968.

18. A. Al-Muqrin, A. El-Sharkawy, and W. M. Abdellah, "Uranium content in groundwater by laser fluorimetry; method validation and dose assessment,” J. Radiol. Prot., vol. 38, no. 3, p. 1140, 2018. 